\title{
ON FINITE ELATION GENERALIZED QUADRANGLES
} WITH SYMMETRIES

\author{
DIRK HACHENBERGER
}




\title{
ON FINITE ELATION GENERALIZED QUADRANGLES WITH SYMMETRIES
}

\author{
DIRK HACHENBERGER
}

\begin{abstract}
We study the structure of finite groups $G$ which act as elation groups on finite generalized quadrangles and contain a full group of symmetries about some line through the base point. Such groups are related to the translation groups of translation transversal designs with parameters depending on those of the quadrangles.

Using results on the structure of $p$-groups which act as translation groups on transversal designs and results on the index of the Hughes subgroups of finite $p$-groups, we can show how restricted the structure of elation groups of finite generalized quadrangles with symmetries is.

One of our main results is that $G$ is necessarily an elementary abelian 2-group, provided that $G$ has even cardinality. In particular, the elation generalized quadrangle coordinatized by $G$ is a translation generalized quadrangle with $G$ as translation group, that is, $G$ contains full groups of symmetries about every line through the base point.
\end{abstract}

\section{Elation generalized quadrangles and 4-gonal families}

The general background of this paper is the study of finite geometries admitting a particular group of automorphisms such that the geometry under consideration can be coordinatized by that group, and, where the properties of the geometry are reflected in the subgroup structure of the automorphism group. Indeed, in the cases considered here, the existence of such a geometry together with its automorphism group can equivalently be formulated as a combinatorial problem concerning the subgroup structure of the coordinatizing group. Therefore, nonexistence results and examples of these geometric structures can be obtained by considering a pure group theoretic problem.

In the present paper, we study the structure of finite groups admitting certain families of subgroups having 'extreme intersection properties' (see the conditions (K1) and (K2) below). The motivation therefore is due to the fact that these groups arise as automorphism groups of finite elation generalized quadrangles.

Since their introduction as generalized polygons by J. Tits in [20], finite generalized quadrangles have been studied intensively in the past three decades. The standard reference, which we refer to for details, is the monograph of S. E. Payne and J. A. Thas [16]. Here we give only the definition of the particular geometry we are interested in, together with its automorphism group.

Let $s, t \geqslant 1$ be integers. A finite generalized quadrangle $\mathscr{Q}$ of type $(s, t)$ is a triple $(\mathscr{P}, \mathscr{L}, \mathscr{I})$, where $\mathscr{P}$ and $\mathscr{L}$ are nonempty and disjoint sets whose elements are called points and lines, respectively, and where $\mathscr{I}$ is a subset of the cartesian product $\mathscr{P} \times \mathscr{L}$, the incidence relation, satisfying the following conditions.

(GQ1) For any $P$ in $\mathscr{P}$, the set $\mathscr{L}_{P}:=\{l \in \mathscr{L}:(P, l) \in \mathscr{I}\}$ has cardinality $t+1$.

(GQ2) For any $l$ in $\mathscr{L}$, the set $\mathscr{P}_{l}:=\{P \in \mathscr{P}:(P, l) \in \mathscr{I}\}$ has cardinality $s+1$.

Received 31 May 1994.

1991 Mathematics Subject Classification 51E12.

J. London Math. Soc. (2) 53 (1996) 397-406 
(GQ3) For any two different points $P$ and $Q$ in $\mathscr{P}$, the cardinality of $\mathscr{L}_{P} \cap \mathscr{L}_{Q}$ is at most onc.

(GQ4) For any two different lines $l$ and $m$ in $\mathscr{L}$, the cardinality of $\mathscr{P}_{l} \cap \mathscr{P}_{m}$ is at most one.

(GQ5) For any pair $(P, l)$ in $\mathscr{P} \times \mathscr{L}$ which is not contained in $\mathscr{I}$, there exists exactly one pair $(Q, m)$ in $\mathscr{I}$ such that $(P, m)$ and $(Q, l)$ are members of $\mathscr{I}$.

A collineation of $\mathscr{Q}$ is a pair $(\alpha, \beta)$, where $\alpha$ and $\beta$ are permutations of the sets $\mathscr{P}$ and $\mathscr{L}$, respectively, such that the incidence relation is respected, that is, $(P, l) \in \mathscr{I}$ if and only if $(\alpha(P), \beta(l)) \in \mathscr{I}$. It is customary to denote a collineation by a single letter.

If $\theta$ is a collineation of $\mathscr{Q}$ which fixes a point $P$ and any line incident with $P$, then $\theta$ is called a whorl about $P$. A whorl $\theta$ about $P$ is called an elation about $P$ if $\theta$ is the identity on $\mathscr{Q}$ or $\theta$ fixes no point of the set $\mathscr{P}-P^{\perp}$, where $P^{\perp}:=$ $\left\{X \in \mathscr{P}: \mathscr{L}_{P} \cap \mathscr{L}_{X} \neq \varnothing\right\}$ is the set of points which have a line in common with $P$.

A finite generalized quadrangle is called a finite elation generalized quadrangle with elation group $G$ and base point $P$, and is denoted by $\left(\mathscr{2}^{(P)}, G\right)$, if $G$ is a group of elations about $P$ acting sharply transitively on the set $\mathscr{P}-P^{\perp}$.

From now on, let $\left(\mathscr{Q}^{(P)}, G\right)$ be a finite elation generalized quadrangle of type $(s, t)$, where $s, t>1$. We are interested in the structure of the elation group $G$, and therefore summarize the fact (due to W. M. Kantor) that $G$ necessarily admits certain families of subgroups having extreme intersection properties (see, for example, [16, Section 8.2]).

Let $X$ be any point in $\mathscr{P}-P^{\perp}$. If $l$ is any line in $\mathscr{L}_{P}$ then $l$ is not incident with $X$ (by the definition of $P^{\perp}$ ) and therefore, by (GQ5), there exists a unique pair $(Y, m)$ in $\mathscr{I}$ such that $(Y, l)$, and $(X, m)$ are elements of $\mathscr{I}$. Hence there are defined two mappings $\phi: \mathscr{L}_{P} \rightarrow \mathscr{L}_{X}$ and $\varphi: \mathscr{L}_{P} \rightarrow P^{\perp} \cap X^{\perp}$, which by (GQ5) are bijections.

Now, for any $l$ in $\mathscr{L}_{P}$, let $A_{l}$ be the stabilizer of the line $\phi(l)$ in $G$, and let $A_{l}^{*}$ be the stabilizer of the point $\varphi(l)$ in $G$. Then $A_{l}$ and $A_{l}^{*}$ are subgroups of $G$ of order $s$ and $s t$, respectively, and furthermore, $A_{l}$ is a subgroup of $A_{l}^{*}$. Moreover, $\mathscr{F}:=\left\{A_{l}: l \in \mathscr{L}_{P}\right\}$ and $\mathscr{F}^{*}:=\left\{A_{l}^{*}: l \in \mathscr{L}_{P}\right\}$ are families of $t+1$ subgroups of $G$, satisfying the following conditions:

(K1) $A B \cap C=\{1\}$ for pairwise distinct $A, B, C$ in $\mathscr{F}$;

(K2) $A^{*} \cap B=\{1\}$ for distinct $A, B$ in $\mathscr{F}$.

The pair $\left(\mathscr{F}, \mathscr{F}^{*}\right)$ is called a 4-gonal family of type $(s, t)$ in $G$.

It was first shown by Kantor (see [11, Theorem 2]) that, conversely, a group of order $s^{2} t$ admitting a 4-gonal family can be represented as a regular elation group of a suitable generalized quadrangle. A 4-gonal family is therefore also called a Kantor family (see [5, 6]). In [11], it is also shown that many, though not all, of the previously known finite generalized quadrangles are elation generalized quadrangles and therefore constructable via a 4-gonal family in the corresponding elation group. Moreover, in [11], a (at that time) new class of elation generalized quadrangles was constructed. For the discussion of further examples, we refer the reader to the recent survey article [15] of S. E. Payne and to [16, Chapter 10].

Consequently, the existence of finite elation generalized quadrangles is completely settled, if the groups of order $s^{2} t$ admitting a 4-gonal family together with such a family are known. For this reason, D. Frohardt and $X$. Chen in $[\mathbf{5 , 6 ]}$ study the restriction that the existence of a 4-gonal family in a group $G$ imposes on the structure 
of $G$. We state two of their main results which are of interest for our considerations (as already mentioned above, we assume that $s, t>1$, throughout).

THEOREM 1.1. (Frohardt [6]). Let $G$ be a group of order $s^{2} t$ admitting a 4-gonal family of type $(s, t)$. If $t \geqslant s$ then $s$ and $t$ are powers of the same prime number $p$ and $G$ is a p-group.

THEOREM 1.2. (Chen and Frohardt [5]). Let $G$ be a group of order $s^{2} t$ admitting a 4-gonal family $(\mathscr{F}, \mathscr{F} *)$ of type $(s, t)$. If there exist two distinct members in $\mathscr{F}$ which are normal subgroups of $G$, then $s$ and $t$ are powers of the same prime number $p$ and $G$ is an elementary abelian p-group.

In the present paper, we are going to study the structure of groups $G$ admitting a 4-gonal family $\left(\mathscr{F}, \mathscr{F}^{*}\right)$ with the additional property that there exists one member of $\mathscr{F}$ which is a normal subgroup of $G$. The normality of some member in $\mathscr{F}$ has the following geometric meaning (see $[16,8.2 .2$.(iv)]).

For a line $l$ in $\mathscr{L}$, let $l^{\perp}:=\left\{m \in \mathscr{L}: \mathscr{P}_{l} \cap \mathscr{P}_{m} \neq \varnothing\right\}$ be the set of lines which have a point in common with $l$. Now, in the above situation, $A_{l}$ is a normal subgroup of $G$, if and only if $A_{l}$ is a full group of symmetries about $l$, that is, any elation in $A_{l}$ fixes each line $m$ of $l^{\perp}$ and acts sharply transitively on the set $\mathscr{P}_{m}-\left(\mathscr{P}_{l} \cap \mathscr{P}_{m}\right)$ of each such line $m$.

In the next section, we shall see that an elation generalized quadrangle admitting a full group of symmetries about a line through the base point is closely related to some other kind of coset geometries, likewise group constructable, and which also have been intensively studied in the past few years, namely translation transversal designs. Using results on the structure of the translation group of such a design, we draw conclusions on the structure of an elation group with symmetries of a generalized quadrangle. A detailed outline of our results is given subsequently to Theorem 2.1. The following theorem is a short summary.

THEOREM 1.3. Let $G$ be a finite group of order $s^{2} t$ admitting a 4-gonal family $\left(\mathscr{F}, \mathscr{F}{ }^{*}\right)$ of type $(s, t)$. If there exists a member $A$ in $\mathscr{F}$ which is a normal subgroup of $G$, then $s$ and $t$ are powers of the same prime number $p$, and necessarily one of the following two cases occurs:

(1.3.1) $G$ is elementary abelian;

(1.3.2) $p$ is odd, $G / A$ is nonabelian and has exponent $p$.

\section{On the structure of regular elation groups with symmetries}

Throughout this section, let $\left(\mathscr{F}, \mathscr{F}^{*}\right)$ be a 4 -gonal family of type $(s, t)$ in a group $G$, which will be written multiplicatively. We assume that there exists a member $A$ in $\mathscr{F}$ which is a normal subgroup of $G$ and study the restriction these assumptions impose on the structure of $G$.

First, we consider the set

$$
\mathscr{F}_{A}:=\left\{A^{*} / A\right\} \cup\{B A / A: B \in \mathscr{F}-\{A\}\}
$$

of subgroups of the factor group $G / A$. 
The cardinality of $A^{*} / A$ is equal to $t$ and the cardinality of $B A / A$ is equal to $s$ for all $B$ in $\mathscr{F}-\{A\}$. Furthermore, as a consequence of $(\mathrm{K} 1)$ and $(\mathrm{K} 2)$, we have that $X \cap Y=A / A$ for any two different members $X$ and $Y$ of $\mathscr{F}_{A}$. Moreover, as

$$
\left|\bigcup_{X \in \mathscr{F}_{A}} X\right|=\left|A^{*} / A\right|+\sum_{B \in \mathscr{F}-\{A\}}(|B A / A|-1)=t+t(s-1)=t s=|G / A|
$$

we see that $\mathscr{F}_{A}$ is a so-called $(t, s)$-partition in $G / A$.

A partition of a group $H$ is a set of proper nontrivial subgroups of $H$ whose union is the whole set $H$, and where any two different members intersect in the trivial subgroup $\{1\}$. The members of a partition are called components. The subgroup $A^{*} / A$ of the (particular) partition $\overline{\mathscr{F}}_{A}$ above is called the particular component of $\mathscr{F}_{A}$.

Finite groups admitting a partition were first studied by R. Baer in [1]. In further papers, R. Baer [2,3], O. H. Kegel [12] and M. Suzuki [19] were able to classify all families of finite groups admitting a partition.

Groups admitting a $(t, s)$-partition were intensively studied in the past, because, as with 4-gonal families, they arise as automorphism groups of certain coset geometries.

If $H$ is a group admitting a $(t, s)$-partition $\pi$ with particular component $N$, then the incidence structure

$$
(H,\{U n: U \in \pi-\{N\}, n \in N\}, \epsilon)
$$

is a translation transversal design with translation group $H$.

Using the Classification Theorem of Baer, Kegel and Suzuki, R. H. Schulz [17] and M. Biliotti and G. Micelli [4] classified all families of groups which arise as translation groups on translation transversal designs. For details and further references, the reader is referred to Hachenberger [7]. There, the author gives an elementary proof of the classification of the families of groups admitting a $(t, s)$ partition without using the theorem of Baer, Kegel and Suzuki, and studies the structure of $p$-groups admitting such a partition.

In the present paper, applying results of [7], we draw conclusions on the structure of elation groups $G$ with symmetries of generalized quadrangles. Together with Frohardt's Theorem 1.1, we can give a first approximation of the structure of $G$.

THEOREM 2.1. Let $G$ be a group of order $s^{2} t$ admitting a 4-gonal family $(\mathscr{F}, \mathscr{F} *)$ of type $(s, t)$ and assume that there is a member $A$ in $\mathscr{F}$ which is a normal subgroup of $G$. Then the following hold:

(2.1.1) $A^{*} / A$ is a normal subgroup of $G / A$;

(2.1.2) $t \geqslant s$, and, $s$ and $t$ are powers of the same prime number $p$, and $G$ is a $p$ group;

(2.1.3) every member of $\mathscr{F}-\{A\}$ is elementary abelian;

(2.1.4) with $p$ by (2.1.2) being the unique prime divisor of the cardinality of $G$, the $\mathrm{H}_{p}$-subgroup of $G / A$ (that is, the subgroup of $G / A$ which is generated by all elements of $G / A$ whose order is different from $p$ ) is a subgroup of the particular component $A^{*} / A$ of the corresponding $(t, s)$-partition $\mathscr{F}_{A}$.

Proof. The first statement is the content of [7, Proposition 1.6] which states that the particular component of a $(t, s)$-partition always is a normal subgroup. It likewise can be derived from [5, Lemma 1], which states that $A^{*}$ is a normal subgroup of $G$, provided that $A$ is normal in $G$.

The second statement is [5, Lemma 2]. It is based on Frohardt's Theorem 1.1. 
The third statement follows immediately from [7, Theorem 3.4], which states that in a $(t, s)$-partition of a $p$-group, every component different from the particular one is elementary abelian.

The last statement is formulated as Result (3.1) in [7].

From now on, let $p$ denote the unique prime divisor of the cardinality of $G$. As a consequence of Theorem 2.1 there remain three possibilities for the factor group $G / A$.

Case 1: $G / A$ is elementary abelian.

Case 2: the $\mathrm{H}_{p}$-subgroup of $G / A$ is different from $A / A$.

Case 3: $G / A$ is not elementary abelian and the $\overline{\mathrm{H}}_{p}$-subgroup of $G / A$ is equal to $A / A$. This is equivalent to $G / A$ being nonabelian of exponent $p$.

We next summarize our main results which hold under the general assumptions of this section.

In Case 1 , we prove that $G$ is elementary abelian provided that $G / A$ is elementary abelian (see Theorem 2.3 below). As a corollary we obtain the inequality $t \geqslant s p$ provided that $G$ is not abelian.

In Case 2, we are confronted with the famous Hughes Problem concerning the index of the $\mathrm{H}_{p}$-subgroup in $G$. The $\mathrm{H}_{p}$-subgroup of a group is named after D. R. Hughes, who conjectured that the $\mathrm{H}_{p}$-subgroup of a finite group $K$ is either trivial, equal to $K$, or has index $p$ in $K$ (see [9]).

Although this conjecture holds for many families of finite groups, it is not true in general. For more information on the Hughes Problem, the reader is referred to E. I. Khukhro [13], a recent monograph on nilpotent groups and their automorphisms. However, in Case 2, applying (2.1.4), we know that the index of the Hughes subgroup of $G / A$ is at least equal to $s$, since $A^{*} / A$ has index $s$ in $G / A$. Applying recent results on the index of the $\mathrm{H}_{p}$-subgroup of a $p$-group in combination with Higman's Inequality (see the remark before Theorem 2.5), we are able to exclude Case 2 (see Theorem 2.7 below).

In Theorem 2.5, we show that $G$ is elementary abelian, provided that the parameter $s$ is equal to $p$. Applying this result together with the validity of the Hughes conjecture for finite 2-groups to the special case where $p=2$, we can prove the result mentioned in the Abstract, that is, that under the general assumptions, $G$ is elementary abelian provided it has even order (see Theorem 2.6). In the language of geometry this can be stated as follows.

A finite elation generalized quadrangle with elation group $G$ of even order containing a full group of symmetries about some line through the base point, already contains full groups of symmetries about every line through the base point.

A quadrangle of the latter type is called a translation generalized quadrangle with translation group $G$ (see [16, Section 8.2]). In various places, we shall use the fact that an abelian group admitting a 4-gonal family is necessarily elementary abelian (see [6, p. 145, Corollary]). Obviously, this also follows from Chen and Frohardt's Theorem 1.2, but it is also proved in [16, Chapter 8], where it is shown that the translation group of a translation generalized quadrangle is elementary abelian.

Of course, in Case 3, $p$ is necessarily odd. However, we cannot say more in that case, because, apart from the remarks in [7, (3.9)] not much is known about nonabelian $p$-groups of exponent $p$ admitting a $(t, s)$-partition. Even less is known about the parameters of such a partition. This is a field for further research where new examples of nonabelian groups admitting 4-gonal families may be found. On the 
other hand, as far as the author is aware, no known finite elation generalized quadrangle with nonabelian elation group admits a full group of symmetries about some line through the base point. This might also be an indication for the nonexistence of such quadrangles.

In order to handle Case 1, we prove the following proposition, which is also of individual interest. It states that $\mathscr{F} *$ does not contain too many abelian members, provided that $G$ is not abelian. Here $|U|$ denotes the cardinality of the set $U$.

Proposition 2.2. Let $(\widetilde{F}, \widetilde{F} *)$ be a 4-gonal family of type $(s, t)$ in a group $G$. Let $\mathrm{Z}(G)$ denote the centre of $G$ and for any $B$ in $\mathscr{F}$, let $\zeta_{B}:=|\mathrm{Z}(G) \cap B|$ and $\zeta_{B^{*}}:=\left|Z(G) \cap B^{*}\right|$. Furthermore, let $\mathscr{A}^{*}$ be the subset of members of $\mathscr{F}^{*}$ which are abelian and let $\mathscr{A}:=\left\{B \in \mathscr{F}: B^{*} \in \mathscr{A}^{*}\right\}$. Then the following hold.

(2.2.1) If $B^{*}$ and $C^{*}$ are different members of $\mathscr{A}^{*}$, then $B^{*} \cap C^{*}$ is a subgroup of $\mathrm{Z}(G)$. Moreorer,

$$
\frac{\zeta_{B^{*}}}{\zeta_{B}}=\frac{\zeta_{C^{*}}}{\zeta_{C}}=t \quad \text { and } \quad|\mathrm{Z}(G)|=\zeta_{B} \cdot \zeta_{C} \cdot t
$$

(2.2.2) If the cardinality of $\mathscr{A}$ is at least 3 , then $\zeta_{B}$ is constant for all $B$ in $\mathscr{A}$, say $\sigma$, and $|\mathrm{Z}(G)|=\sigma^{2} t$. Moreover, if $G$ is not elementary abelian, then $\sigma<s$ and the cardinality of $\mathscr{A}$ is at most $s / \sigma+1$, and, in the case of equality, $G / Z(G)$ is necessarily elementary abelian.

Proof. If $G$ is a group containing two abelian subgroups $U$ and $V$ such that $G=U V$, then $\mathrm{Z}(G)=(U \cap \mathrm{Z}(G))(V \cap \mathrm{Z}(G))$.

Let $z=u v$ be an element of the centre of $G$, where $u \in U$ and $v \in V$. Since $V$ is abelian, we have that $u=z v^{-1}$ centralizes $V$. Since $U$ likewise is abelian, we have that $u$ also centralizes $U$ and therefore the whole group $G$, which by assumption is equal to the complex product of $U$ with $V$. Thus $u$ is an element of the centre of $G$. The same argument shows that $v \in \mathrm{Z}(G)$, whence the desired factorization of $\mathrm{Z}(G)$ is proved.

If we apply this argument to any two different members $B^{*}$ and $C^{*}$ of $\mathscr{A}^{*}$, we obtain that $\mathrm{Z}(G)=\left(B^{*} \cap \mathrm{Z}(G)\right)\left(C^{*} \cap \mathrm{Z}(G)\right)$. Furthermore, it is clear that $K:=B^{*} \cap C^{*}$ is a subgroup of $Z(G)$. Now, since $K$, as a consequence of the definition of a 4-gonal family, has cardinality $t$, we obtain

$$
|\mathrm{Z}(G)|=\left(\zeta_{B^{*}} \cdot \zeta_{C^{*}}\right) / t
$$

On the other hand, applying the above argument to any pair $\left(B, C^{*}\right)$, where $B$ and $C$ are different members of $\mathscr{A}$, we have $\mathrm{Z}(G)=(B \cap \mathrm{Z}(G))\left(C^{*} \cap \mathrm{Z}(G)\right)$ and therefore, by $(\mathrm{K} 2),|\mathrm{Z}(G)|=\zeta_{B} \zeta_{C *}$. From that we derive the first statement of the theorem.

Assume next that the cardinality of $\mathscr{A}^{*}$ is at least 3 and let $B, C$ and $D$ be three pairwise different members of $\mathscr{A}$. An application of the first statement to the pairs $\left(B^{*}, C^{*}\right),\left(B^{*}, D^{*}\right)$ and $\left(C^{*}, D^{*}\right)$ shows that

$$
|\mathrm{Z}(G)| / t=\zeta_{B} \cdot \zeta_{C}=\zeta_{B} \cdot \zeta_{D}=\zeta_{C} \cdot \zeta_{D},
$$

which proves the first part of the second statement.

Now, let $\sigma$ be as in (2.2.2). If $G$ is not elementary abelian, then (by Theorem 1.2) $G$ is not abelian, whence $\sigma<s$. In that case,

$$
\mathscr{A}_{Z(G)}:=\{B Z(G) / Z(G): B \in \mathscr{A}\}
$$


is a set of proper subgroups of $G / \mathrm{Z}(G)$. Moreover, if $X$ and $Y$ are different members of $\mathscr{A}_{\mathrm{Z}(G)}$, an argument similar to the one used at the very beginning of the proof shows that $X$ and $Y$ intersect in the trivial subgroup $\mathrm{Z}(G) / \mathrm{Z}(G)$. Thus, it is easy to see that $\mathscr{A}_{Z(G)}$ is an $(s / \sigma,|\mathscr{A}|)$-partial congruence partition in $G / Z(G)$, that is, a set of $|\mathscr{A}|$ subgroups of $G / Z(G)$ having the property that any two different members have intersection $\mathrm{Z}(G) / Z(G)$ while their complex product is equal to $G / Z(G)$. Therefore, the cardinality of $\mathscr{A}$ is at most $s / \sigma+1$, and equality occurs if and only if $\mathscr{A}_{Z(G)}$ is a partition of $G / Z(G)$. Such a partition is called a congruence partition in $G / Z(G)$. A well-known theorem on congruence partitions says that finite groups admitting such a partition are necessarily elementary abelian (we refer the reader to Chapter 1 of $\mathrm{H}$. Lüneburg's monograph [14]; translation planes are the geometric equivalent to congruence partitions). This finally proves (2.2.2).

We can now prove that Case 1 only occurs, if $G$ is elementary abelian.

THEOREM 2.3. Let $G$ be a group of order $s^{2} t$ admitting a 4-gonal family $\left(\mathscr{F}, \mathscr{F}^{*}\right)$ of type $(s, t)$. Assume that there exists a member $A$ in $\mathscr{F}$ which is a normal subgroup of $G$ and suppose that $G / A$ is elementary abelian. Then $G$ is elementary abelian.

Proof. If $A$ is a member of $\mathscr{F}$ which is normal in $G$, and $G / A$ is elementary abelian, then $B^{*}$ is elementary abelian for all $B^{*}$ in $\mathscr{F}^{*}-\left\{A^{*}\right\}$, since $B^{*}$ as a complement of $A$ (see $(\mathrm{K} 2)$ ) is isomorphic to $G / A$. We may therefore apply Proposition 2.2 to $\mathscr{A}^{*}:=\mathscr{F}^{*}-\left\{A^{*}\right\}$.

If $|\mathscr{F}|>3$, that is, $\left|\mathscr{A}^{*}\right| \geqslant 3$, let $\sigma$ be as in (2.2.2). Assume that $G$ is not elementary abelian. Then $G$ is not abelian by Theorem 1.2, whence $\sigma<s$ and thus, by (2.2.2), $t=\left|\mathscr{A}^{*}\right| \leqslant s / \sigma+1$. But, as $t \geqslant s$, see (2.1.2), this can happen only if $\sigma=1$. Consequently, by the definition of $\sigma$ and Proposition 2.2, the centre of $G$ is contained in $B^{*}$ for all $B$ which are different from $A$. Therefore, by (K2), $A$ intersects $Z(G)$ in the trivial subgroup $\{1\}$. But this is a contradiction, since any normal subgroup $X \neq\{1\}$ of a $p$-group has nontrivial intersection with $\mathrm{Z}(G)$. Thus, $G$ is elementary abelian.

In order to complete the proof of the theorem, it remains to study the case, where $|\mathscr{F}|=3$, that is, where $t=2$. As $s>1$ by assumption and $t \geqslant s$ by $(2.1 .2)$, we see that $s=t=2$ and therefore $|G|=8$. Hence, any $B^{*}$ in $\mathscr{F}^{*}$ is a maximal subgroup of $G$ and therefore normal in $G$. As by assumption $A$ is normal in $G$, (K2) implies that $G$ is isomorphic to the direct product of $A$ with $B^{*}$, where $B$ is any element of $\mathscr{F}$ which is different from $A$. But then $G$ is abelian, since $|A|=2$ and $\left|B^{*}\right|=4$. Finally, Theorem 1.2 implies that $G$ is elementary abelian.

COROLLARY 2.4. Let $G$ be a finite p-group admitting a 4-gonal family ( $\mathscr{F}, \mathscr{F} *)$ of type $(s, t)$ and suppose there is a member $A$ in $\mathscr{F}$ which is a normal subgroup of $G$. If $G$ is not abelian, then necessarily $t \geqslant s p$.

Proof. By (2.1.2), we know that $t \geqslant s$. If $t=s$, we have that the corresponding $(s, s)$-partition in $G / A$ is a congruence partition in $G / A$. Therefore (compare with the end of the proof of Proposition 2.2) $G / A$ is elementary abelian. By Theorem 2.3, in contradiction to our assumption, we obtain that $G$ is elementary abelian. Thus, $t>s$, and, as $t$ and $s$, are powers of $p$, we have the desired result. 


\section{References}

1. R. BAER, 'Partitionen endlicher Gruppen', Math. Z. 75 (1961) 333-372.

2. R. BAER, 'Einfache Partitionen endlicher Gruppen mit nicht-trivialer Fittingscher Untergruppe', Arch. Math. (Basel) 12 (1961) 81 - 89.

3. R. BAER, 'Einfache Partitionen nicht-einfacher Gruppen', Math. Z. 77 (1961) 1-37.

4. M. Biliotti and G. Micelli, 'On translation transversal designs', Rend. Sem. Mat. Univ. Padova 73 (1985) 217-229.

5. X. Chen and D. Frohardt, 'Normality in a Kantor-family', J. Combin. Theory Ser. A 64 (1993) $130-136$.

6. D. Frohard, 'Groups which produce generalized quadrangles', J. Combin. Theory Ser. A 48 (1988) $139-145$.

7. D. Hachenberger, 'Remarks on translation transversal designs', J. Algebra 166 (1994) $211-231$.

8. D. R. Hughes, 'Partial difference sets', Amer. J. Math. 78, (1956) 650-674.

9. D. R. Hughes, 'A problem in group theory', Bull. Amer. Math. Soc. 63 (1957) 209.

10. B. HuPPERT, Endliche Gruppen I (Springer, Berlin, 1967).

11. W. M. Kantor, 'Generalized quadrangles associated with $G_{2}(q)$ ', J. Combin. Theory Ser. A 29 (1980) 212-219.

12. O. H. KeGEL, 'Nichteinfache Partitionen endlicher Gruppen ', Arch. Math. (Basel) 12 (1961), 170-175.

13. E. I. KHukHro, Nilpotent groups and their automorphisms (de Gruyter, Berlin-New York, 1993).

14. H. LÜNEBURG, Translation planes (Springer, Berlin-Heidelberg-New York, 1980).

15. S. E. PAYNE, 'A census of finite generalized quadrangles', Finite geometries, buildings and related topics (eds. W. M. Kantor, R. A. Liebler, S. E. Payne, E. E. Shult; Clarendon Press, Oxford, 1990) 29-36.

16. S. F. PAyne and J.A. Thas, Finite generalized quadrangles Pitman Research Notes in Mathematics Series 110 (Longman Sci. Tech., Boston, 1984).

17. R.-H. Schulz, 'On the classification of translation group-divisible designs' European J. Combin. 6 (1985) 369-374.

18. E. G. Strals and G. Szekeres, 'On a problem of D. R. Hughes', Proc. Amer. Math. Soc. 9 (1958) $157-158$.

19. M. Suzuk!, 'On a finite group with a partition', Arch. Math. (Basel) 12 (1961) 241-254.

20. J. Tits, 'Sur la trialité et certain groupes qui s'en déduisent', Inst. Hautes Études Sci. Publ. Math. 2 (1959) 14-60.

Institut für Mathematik der Universität Augsburg

Universitätsstraße 14

D-86135 Augsburg

E-mail: Hachenberger@math.uni-augsburg.de 